\title{
Label-Free Shape-Based Selection of Cardiomyocytes with on-Chip Imaging Cell Sorting System
}

\section{Fumimasa Nomura, Tomoyuki Kaneko, Akihiro Hattori and Kenji Yasuda*}

Department of Biomedical Information, Institute of Biomaterials and Bioengineering, Tokyo Medical and Dental University, 2-3-10 Kanda-Surugadai, Chiyoda, Tokyo 1010062, Japan

\begin{abstract}
We have examined the method for the label-free optical microscopic image-based selection of cardiomyocytes from the mixture of collagenase-digested embryonic mouse heart cells using the on-chip imaging cell sorter system, in which 1/2000 s real-time high-speed camera-based phase-contrast cell image recognition was performed. To separate the cardiomyocytes from other cells including fibroblasts, we distinguished the roughness of the cell surface quantitatively as the index of sorting. The selected cells with smooth surface (roughness index not more than 1.1) for cardiomyocytes, and confirmed that the more than $80 \%$ of the collected and recultivated cells were self-beating and the $99.2 \%$ of them were identified as cardiomyocytes by immunostaining. The results indicates the potential of the labelfree cell purification using imaging cell sorting system, especially for cardiomyocytes by the index of their cell surface roughness, which might be applicable for purification of cardiomyocytes for regenerative medicine.
\end{abstract}

Keywords: On-chip imaging cell sorting system, Cardiomyocyte, Index of roughness, Agarose microchamber

\section{Introduction}

The non-invasive purification technique of the particular target cells from tissues, organs or cultivated cells is the essential and fundamental for cell-based research and even for regenerative medicine. For example, for the purification of cardiomyocytes, we need to remove more than half of the noncardiomyocytes from whole heart cells [1].

At present, the most commonly used method for cardiomyocyte purification is the pre-cultivation method exploiting the difference of the adhesion of cardiomyocytes and other cells including fibroblasts to the surface of cultivation dish [2]. However, the method was probabilistic way and is still regarded to be a rough purification. Another more precise purification method is the fluorescence-activated cell sorting (FACS) [3] with fluorescent cardiomyocyte marker labeling, whereas it involves the potential problems of cell damages caused by antibody labeling. To overcome this labeling problem, we have proposed and developed the on-chip imaging cell sorting system for the label-free phase-contrast imaging-based identification of cell types using the index of cell surface shapes. Different shapes with $1 / 2000$ s real time processing $[4,5]$. In this paper, as a practical application of imaging cell sorting, we have examined the purification efficacy of cardiomyocytes cells with using of the index of cell surface roughness and reported.

\section{Materials and Methods \\ Imaging cell sorter system}

The on-chip phase-contrast imaging cell sorter system was consisted of the following five modules; [4, 5] a cell sorting chip, an optical microscope equipped with a phase-contrast image processing module, a 1/2000 s high-speed charge-coupled devise (CCD) camera (HAS-220; DITECT, Japan), image processing unit, and a computercontrolled switching DC power supply for cell separation (Figure 1a). The cell sorting chip was made of poly dimethylsiloxane (PDMS) attached on a $0.9 \mathrm{~mm}$ thin glass slide, in which two inlets and flow channels for sample solution and buffer solution were symmetrically arranged. The generated two laminar flow in the two channels were connected at the cell separation area in center of the chip, and the two laminar flows were divided to the symmetrical two flow channels again, one is for waste drain and the other is for target cell collection. A pair of $1.5 \%$ agarose gel electrodes containing $0.5 \mathrm{M} \mathrm{NaCl}$ (carrier of ionic current) was also arranged at the connecting point of two laminar flows for cell separation to shift target cells from the sample laminar flow to the buffer laminar flow through their boundary by electrophoretic force.

First for separation of target cells from the mixture of cells, the samples were applied to the sample inlet (Figure 1b) and put the air pressure both to the sample inlet and the buffer inlet to introduce the cells into the central separation area with keeping the boundary of two laminar flows by maintaining the same flow velocities of medium buffers (Figure 1c). The phase-contrast real-time images of the cells flew in the sample flow channel were directly monitored with 2000 pictures/s intervals, and were analyzed to acquire specific indexes (cell size, cross-sectional area, cell surface roughness) of cells using the fieldprogrammable gate array (FPGA) real time image processing unit. The system can record both of the cell image pictures and FPGA analysis data simultaneously for the further checking and evaluation.

Then, when the target cells which satisfy the required values of the indexes were recognized, they were sorted by shifting them from left laminar flow to right laminar flow by the computer-controlled electrostatic force generation between two gel electrodes, which arranged perpendicular to the flow direction at the separation area, by the switching DC power supply.

Finally, only the recognized and shifted target cells were moved

*Corresponding author: Kenji Yasuda, Department of Biomedical Information, Institute of Biomaterials and Bioengineering, Tokyo Medical and Dental University 2-3-10 Kanda-Surugadai, Chiyoda, Tokyo 101-0062, Japan E-mail: yasuda.bmi@ tmd.ac.jp

Received September 14, 2011; Accepted December 05, 2011; Published December 07, 2011

Citation: Nomura F, Kaneko T, Hattori A, Yasuda K (2011) Label-Free ShapeBased Selection of Cardiomyocytes with on-Chip Imaging Cell Sorting System. J Bioprocess Biotechniq S3:003 doi:10.4172/2155-9821.S3-003

Copyright: @ 2011 Nomura F, et al. This is an open-access article distributed unde the terms of the Creative Commons Attribution License, which permits unrestricted use, distribution, and reproduction in any medium, provided the original author and source are credited. 
Citation: Nomura F, Kaneko T, Hattori A, Yasuda K (2011) Label-Free Shape-Based Selection of Cardiomyocytes with on-Chip Imaging Cell Sorting System. J Bioprocess Biotechniq S3:003 doi:10.4172/2155-9821.S3-003

Page 2 of 6

from the waste drain channel flow to the target sample collection channel flow, and the target cells were acquired at the target cell reservoir arranged at the end of the target sample collection channel (Figure $1 \mathrm{~b}$ and $1 \mathrm{c}$ ).

\section{Sample preparation}

The cardiomyocytes were isolated from 12- to 13-day-old mouse embryos (ICR) (Saitama Experimental Animals Supply, Japan) [6]. A mouse was anesthetized with diethyl ether and embryos were rapidly removed. The hearts of the embryos were removed and then washed with phosphatebuffered saline (PBS, $137 \mathrm{mM} \mathrm{NaCl}, 2.7 \mathrm{mM} \mathrm{KCl}, 8$
$\left.\mathrm{mM} \mathrm{Na} \mathrm{HPO}_{4}, 1.5 \mathrm{mM} \mathrm{KH} \mathrm{PO}_{4}, \mathrm{pH} 7.4\right)$ containing $0.9 \mathrm{mM} \mathrm{CaCl}$ and $0.5 \mathrm{mM} \mathrm{MgCl}_{2}$ to induce heart contraction and remove corpuscles. The hearts were then transferred to PBS without $\mathrm{CaCl}_{2}$ and $\mathrm{MgCl}_{2}$ and minced into $1-\mathrm{mm}^{3}$ pieces with fine scissors, and incubated in PBS containing $0.25 \%$ collagenase (Wako, Osaka, Japan) for $30 \mathrm{~min}$ at $37^{\circ} \mathrm{C}$ to digest the ventricular tissue. After this procedure was repeated twice, the cell suspension was transferred to a cell culture medium (DMEM [Invitrogen Corp, Carlsbad, SC, USA] supplemented with 10\% fetal bovine serum, $100 \mathrm{U} / \mathrm{ml}$ penicillin, and $0.1 \mathrm{mg} / \mathrm{ml}$ streptomycin) at $4{ }^{\circ} \mathrm{C}$. The cells were filtered through a $40-\mu \mathrm{m}$-nylon mesh and were centrifuged at $180 \mathrm{~g}$ for $5 \mathrm{~min}$ at room temperature. Then, the cell pellet (a) DC power supply

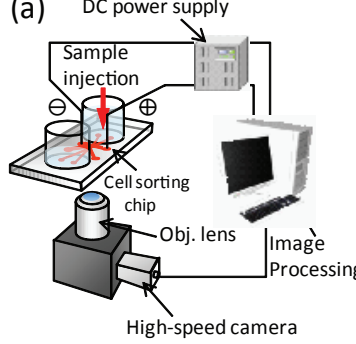

(b)

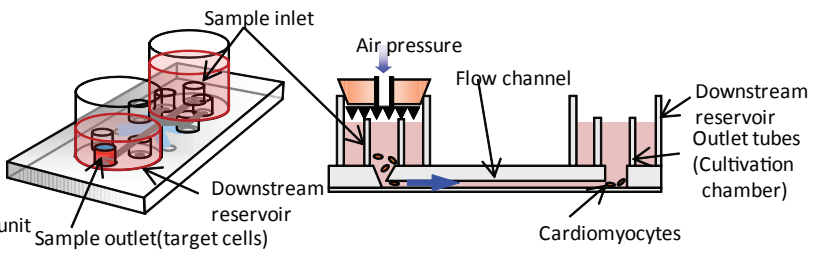

Buffer channel

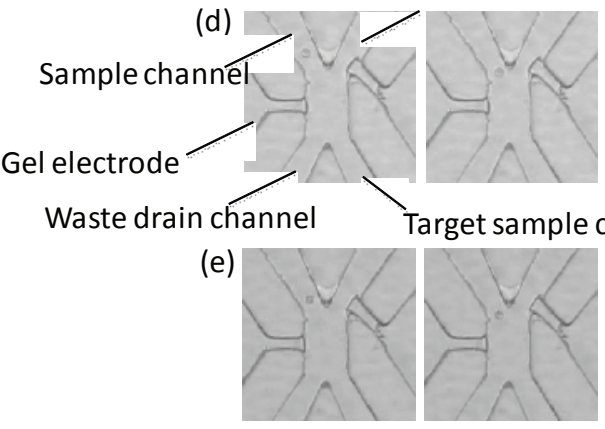

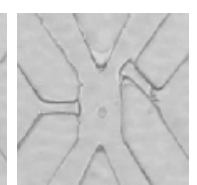
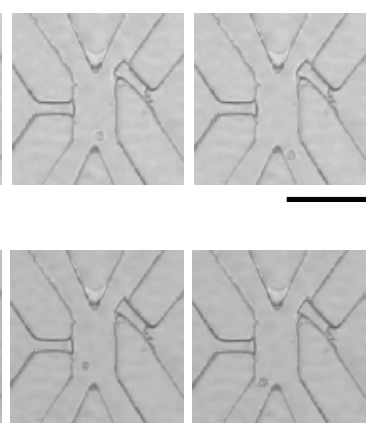

Figure 1: Selection of cardiomyocytes with on-chip cell sorting system.

(a) System design of on-chip cell sorting system. (b) Chip design of on-chip cell sorting system. (c) Cross sectional view of the chip. (d) The time course images of cell sorting area during the collection of selected cells. Each image was obtained $33 \mathrm{~ms}$. Bar, $100 \mu \mathrm{m}$. (e) The time course images of cell sorting area during the removing the non-selected cells. Each image was obtained 33ms. Bar, $100 \mu \mathrm{m}$.

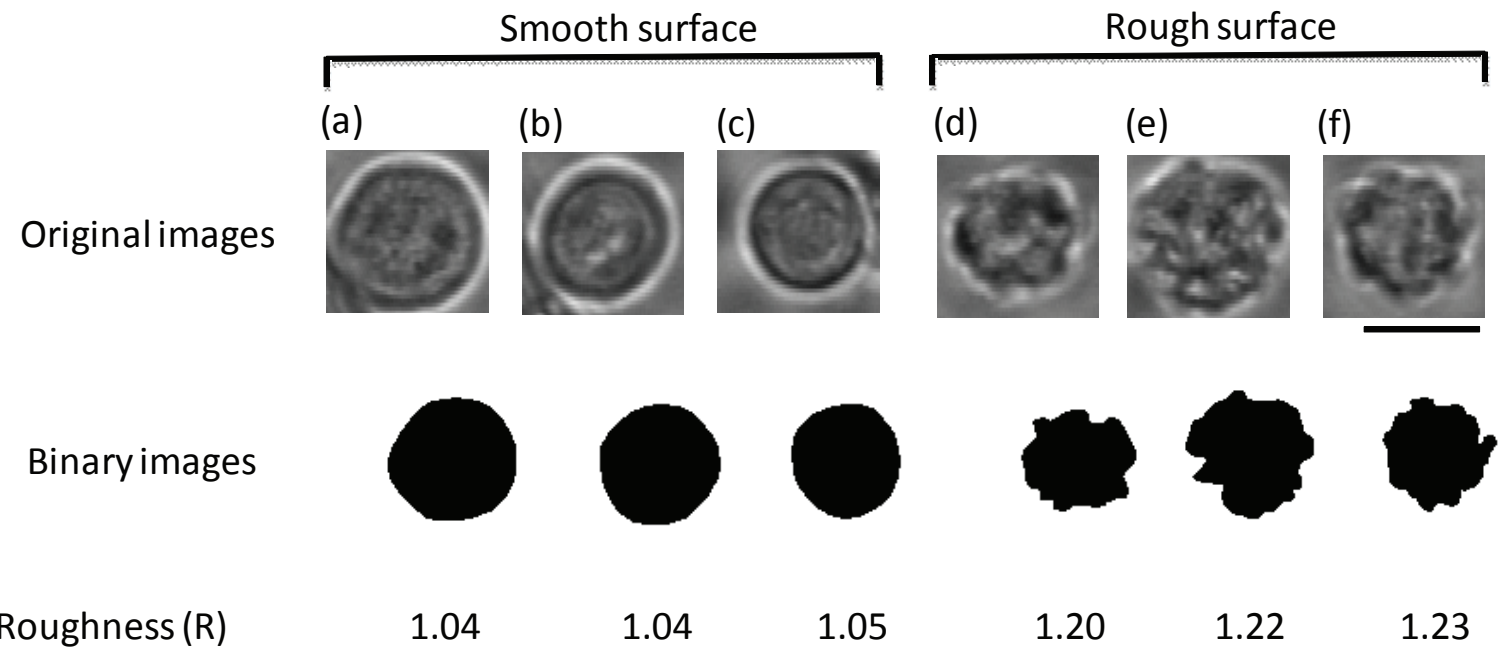

Figure 2: Selection of cardiomyocytes with on-chip cell sorting system.

(a)-(c) Acquired images and sorting indices of smooth surface cells, and (d)-(f) rough surface cells. Bar, $10 \mu \mathrm{m}$. 


\begin{tabular}{|l|l|l|l|l|l|}
\hline $\begin{array}{l}\text { Sample } \\
\text { (pixel) }\end{array}$ & Area (pixel) & $\begin{array}{l}\text { Perimeter } \\
\text { (pixel) }\end{array}$ & $\begin{array}{l}\text { Radius } \\
\text { (pixel) }\end{array}$ & $\begin{array}{l}\text { Circumfer- } \\
\text { ence (pixel) }\end{array}$ & \\
\hline (a) & 4232 & 240.6 & 36.7 & 230.6 & 1.04 \\
\hline (b) & 3203 & 209.1 & 31.9 & 200.6 & 1.04 \\
\hline (c) & 2767 & 195.2 & 29.6 & 186.4 & 1.04 \\
\hline (d) & 2431 & 209.2 & 27.8 & 174.7 & 1.20 \\
\hline (e) & 3787 & 265.0 & 34.7 & 218.1 & 1.21 \\
\hline (f) & 2947 & 236.1 & 30.6 & 192.4 & 1.22 \\
\hline
\end{tabular}

Table 1: Quantitative evaluation of cell surface roughness (Fig. 2).

was resuspended in a cell culture medium and incubated at least $20 \mathrm{~min}$ in room temperature.

\section{Immunostaining of cardiomyocytes}

The cells were fixed with $4 \%$ paraformaldehyde and permeabilized in $0.1 \%$ Triton X-100 and exposed for 2 hours to the mouse monoclonal antibody to heavy chain of cardiac myosin (abcam, Japan) dissolved in blocking buffer (PBS containing 1\% BSA) and incubated for 1 hour at room temperature with Alexa Fluor 546, goat anti-mouse IgG (Molecular probes, Eugene, USA). To visualize the nuclei, cells were counterstained with Hoechst 33342 for $30 \mathrm{~min}$ at room temperature. The cell images were recorded using an inverted microscope equipped for epifluorescence (IX-70, Olympus, Japan) using cooled CCD camera (ORCA-ER, Hamamatsu photonics, Japan).

\section{Results and Discussion}

\section{Index for distinguish the surface roughness}

For the imaging cell sorting, the particular index of cell images is essential for their separation. Regarding separation of cardiomyocytes from the mixture, we have already examined and found that the most of the smooth round shaped cells should cardiomyocytes). Hence, we have defined the index of roughness of cell surface $R$, which compare the real perimeter of cell surface and the estimated perimeter of cell surface by approximation of sphere model, to distinguish the surface roughness of cardiomyocytes and other cells quantitatively as,

$$
R=\frac{l}{\sqrt{4 \pi S}}
$$

Where $l$ is the perimeter of cell surface, $S$ is the cross-sectional area of cell (both of $l$ and $S$ were acquired from pixel-based digitized image analysis of cells).

For example, a sphere with radius $r$ should have $R=[2 \pi r /(4 \pi \times \pi r 2) 1 / 2]$ equal to 1 , and when the roughness increased, $R$ should be increased in proportion to the increase of the real perimeter. Using the above index $R$, we can quantify the smoothness/ roughness of cell surfaces. For example, as shown in Figure 2 and Table 1 , the typical smooth cells have the index of roughness $R$ less than 1.1 (Figures $2 \mathrm{a}, 2 \mathrm{~b}$ and $2 \mathrm{c}$ ), and the typical rough cells have $R$ larger than 1.1 (Figure 2d, 2e, and 2f). Table 1 also shows the acquired values of each cell calculated by the FPGA unit calculation from the captured cell images.

\section{Cardiomyocyte separation using the index of roughness $\mathbf{R}$}

There are two major points for accomplishing the non-labeled separation of cardiomyocytes from the mixture of enzyme-treated heart extracts. One is the development of the proper quantified index of surface roughness of cells for judging the target cells and others as described above, and the other is the proper enzymatical treatment for appearance of surface shape differences to be able to distinguish different cells. Regarding the cardiomyocyte purification, based on our experience, [11] the collagenase-digestion treatment was effective to appear the difference of smooth/rough surface shapes depending on their difference of cell types. Hence, in this experiment, the sample

\section{(a)}

(b)
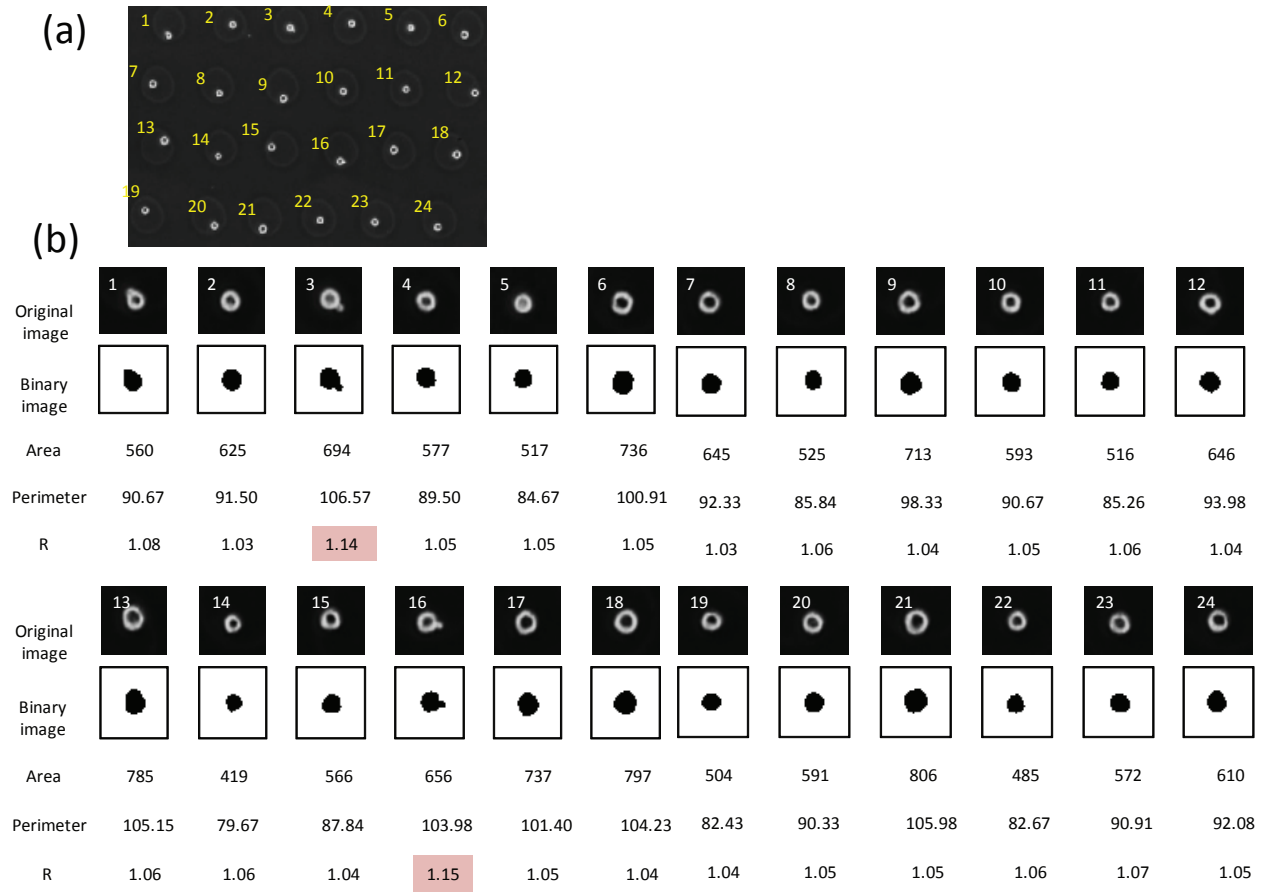

Figure 3: Representative images and indexes of selected smooth surface cells.

(a) Phase-contrast image of arrangement of the selected smooth surface cells settled on agarose microchamber at one by one manner using micropipette after cell sorting purification. (b) Phase-contrast image and FPGA calculated index of cells in Figure 3a. 
Citation: Nomura F, Kaneko T, Hattori A, Yasuda K (2011) Label-Free Shape-Based Selection of Cardiomyocytes with on-Chip Imaging Cell Sorting System. J Bioprocess Biotechniq S3:003 doi:10.4172/2155-9821.S3-003

(a)

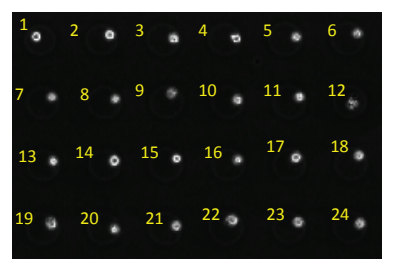

(b)

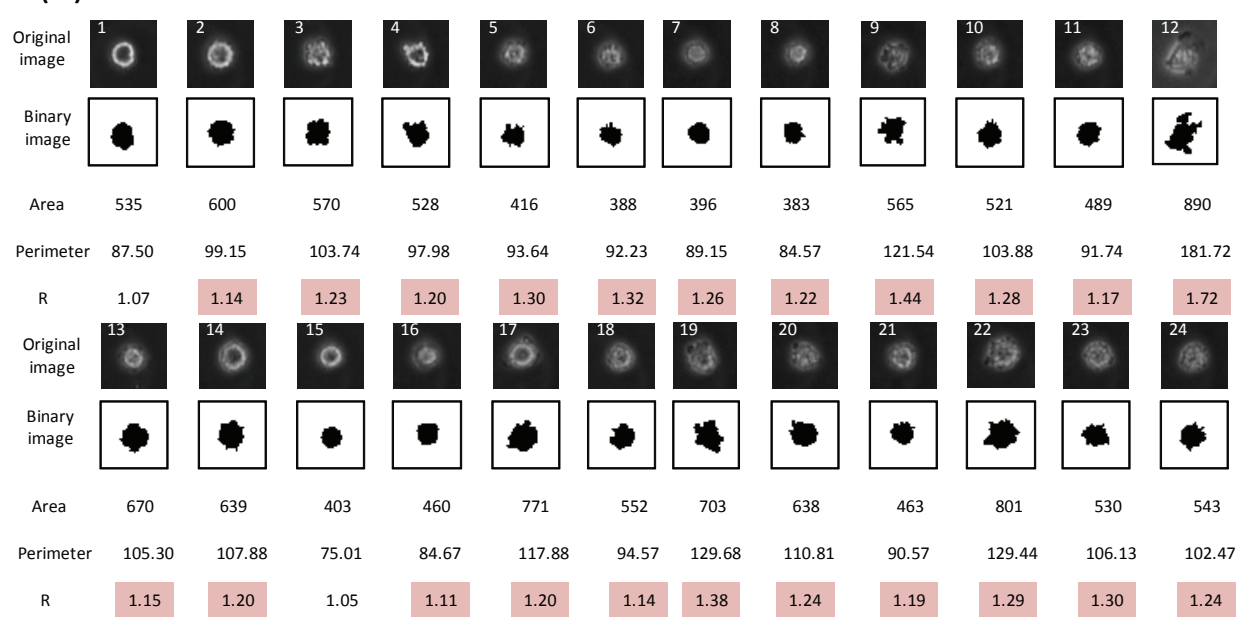

Figure 4: Representative images and indexes of removed rough surface cells.

(a) Phase-contrast image of arrangement of the selected rough surface cells settled on agarose microchamber at one by one manner using micropipette after cell sorting purification. (b) Phase-contrast image and FPGA calculated index of cells in Figure 4a.

$(a)$

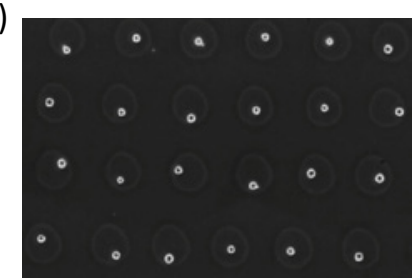

(b)

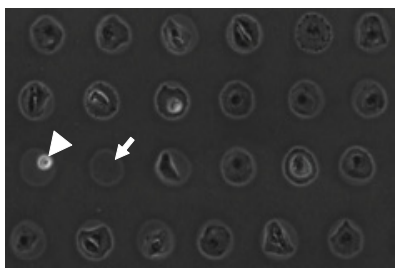

(c)

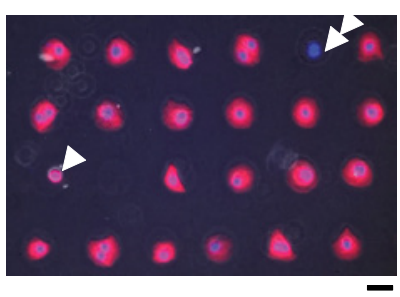

(d)

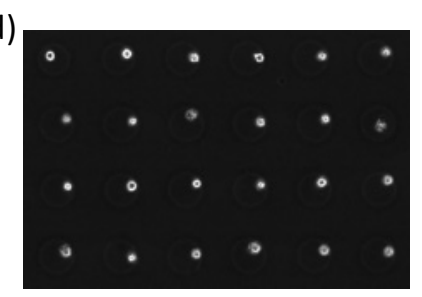

(e)

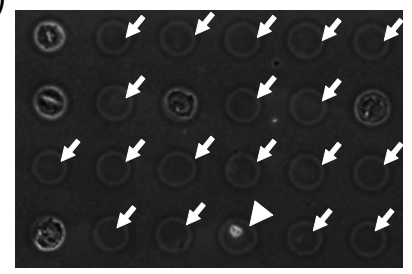

(f)

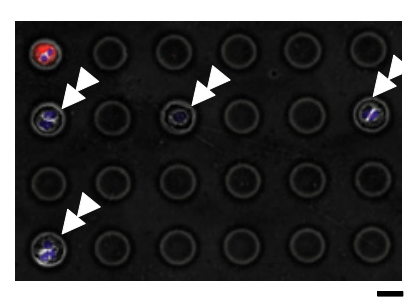

Figure 5. Representative images of selected cells in agarose microchambers.

(a) Phase-contrast image of arrangement of the selected smooth cells on agarose microchamber at one by one manner using micropipette (same image of Figure 3a). (b) Phase-contrast image of selected cells after 2 days cultivation. White arrowhead shows a shrinked cell. White arrow shows the microchamber in which the cell was disappeared during cultivation caused by apoptosis or inufficient attachment. (c) Phase-contrast image superimposed on the fluorescence image of the selected cells ( 2 days cultivation) after fixation with $4 \%$ Formaldehyde solution. Nucleus (Hoechst33342; blue), heavy chain cardiac myosin (red). White double arrowheads show the no-stained cells by cardiac myosin (i.e., non cardiomyocytes). (d) Phase-contrast image of arrangement of the selected rough cells on agarose microchamber at one by one manner using micropipette (same image of Figure 4a). (e) Phase-contrast image of selected cells after 2 days cultivation. White arrowhead shows shrinkage cell. (f) Phase-contrast image superimposed on the fluorescence image of the selected cells (2 days cultivation) after fixation with $4 \%$ Formaldehyde solution. Nucleus (Hoechst33342; blue), heavy chain cardiac myosin (red). Bars, $100 \mu \mathrm{m}$. 


\begin{tabular}{|c|c|c|c|c|c|c|c|}
\hline & 0 DIV & \multicolumn{4}{|c|}{2 DIV } & \multicolumn{2}{|c|}{ staining of cardiac myosin } \\
\hline & selected cell & beating cell & no beating cell & shrinkage cell & removal cell & positive cell & negative cell \\
\hline cell number & 242 & 178 & 19 & 12 & 33 & 195 \\
\hline ratio (\%) & 100 & 73.6 & 7.9 & 5 & 13.6 & 80.6 \\
\hline
\end{tabular}

Table 2: Quantitative evaluation of cardiomyocyte ratio

mixture of cells were acquired from collagenase-digested heart isolated from 13-day-old mouse embryos (ICR). It should be noted for this procedure that the collagenase-digestion procedure and following incubation before cell sorting is essential for appearing the difference of smooth/rough surface of cells. Otherwise, if we use the conventional trypsinization procedure for cell preparation, cells do not show apparent smooth/rough surface shape differences. And the incubation after the collagenase-treatment at least $20 \mathrm{~min}$ was also essential for appearing their shape differences. Without this incubation, the shape difference was not sufficiently appeared on the surface of cells.

The functional sorting procedure of cardiomyocytes using above on-chip imaging cell sorter system with this imaging index $R$ was as follows; (1) when the target smooth cells $(R \leq 1.1)$ flew into the sorting area, the target cell was recognized by high-speed camera and a pulse DC voltage was applied between a pair of agarose gel electrodes to shift the target cells from the sample flow channel to the buffer channel to introduce to the target sample collection channel (Figure 1d); (2) on the other hand, when other rough cells $(R>1.1)$ comes into this area, no electrostatic force was applied and the rough cells were introduced into the waste drain channel (Figure 1e).

Each of the collected smooth surface cell in the target cell reservoir was transferred into the 5 - $\mu$ m-thick $2 \%(\mathrm{w} / \mathrm{v})$ agarose (GenePure LowMelt ISC BioExpress) microchamber [7, 8] laid on the collagen coated polystyrene cultivation dish at one by one manner $[9,10]$ using micropipette (Figures 3, 4 and 5), and cultivated in a cell culture medium (Invitrogen DMEM supplemented with $10 \%$ fetal bovine serum, $100 \mathrm{U} / \mathrm{mL}$ penicillin, and $100 \mathrm{mg} / \mathrm{mL}$ streptomycin) at $37{ }^{\circ} \mathrm{C}$ with a humidified atmosphere of $95 \%$ air and $5 \% \mathrm{CO}^{2}$.

Figure 3 shows the 24 samples of the typical target smooth cells acquired from cell sorting system. As shown in Figure 3a, each cell was put into the microchamber to isolate and identification of cell for identification of their characteristics after cultivation. Figure $3 \mathrm{~b}$ shows the micrographs and the FPGA acquired indexes of the collected smooth cells. The index $R$ of all the smooth cells except for the two coagulated smooth cells (see no. 3 and 16) showed less than 1.08. The samples of coagulated smooth cells (No. 3 and 16) were manually acquired from the rough sample cell reservoir to indicate the potential error of the index $R$ for appropriate smooth cells. The results indicate that the visualized micrograph-based identification of smooth cells is suitable match to the index $R<1.1$.

Figure 4 also shows the 24 samples of the typical rough surface cells acquired from cell sorting system. Just same as Figure 3, each cell was put into the microchamber (Figure $4 \mathrm{a}$ ) and indicated the index $R$ larger than 1.1 except for the samples no. 1 and no. 15, which were manually collected from the smooth sample reservoir to indicate the potential error of the index $R$ for appropriate rough cells, too. In the results, some rough surface cells were judged $R<1.1$ using FPGA procedure. One of the possible reasons and possible improvement for overcoming this problem is using of higher magnitude of objective lens for acquiring more precise perimeters.

\section{Evaluation of the index of surface roughness for characterizing cardiomyocytes}

After two days cultivation of the smooth surface cells (Figure 5b) and the rough surface cells (Figure 5e) laid in the agarose microchambers, we first categorized the cultivated cells by their ability of beating, and also by their adhesion abilities such as shrinking and stable expansion. For example, as shown in Figure 5b, a cell at the white arrowhead was shrinking, and a cell at the white arrow was disappeared. Moreover, as shown in Figure $5 c$, after the staining of myosin heavy chain of cells as the index of cardiomyocyte protein, a cell at the white double arrowheads was not stained, whereas all the remaining 21 cells in the 24 microchambers were well cultivated and showed their beating. In contrast, as shown in Figures 5d, 5e and 5f, most of rough surface cells were not attached on the surface of cultivation dish and disappeared (white arrows). Even the attached cells did not stained by myosin heavy chain probes (white double arrowheads) except for the rough surface sample no. 1 , having the index $\mathrm{R}=1.07$.

In the experiment, from all of the 242 smooth cells, beating cells were 178 (73.6\%), no-beating cells were 19 (7.9\%), shrunk cells were 12 (5.0\%), unattached cells were 33 (13.6\%), respectively (Table 2).

As shown in Figure $5 \mathrm{c}$, cardiac myosin positive-staining cells were 195 (99.99\% of healthy cultivated cells; $80.6 \%$ of whole collected smooth cells), and the negative-staining cells were 2 cells $(0.01 \%$ of healthy cultivated cells; $0.8 \%$ of whole cells) (Table 2). That means, even the cells which were not beating were cardiomyocytes except for two cells from 197 cells.

Hence, the results indicated that at least more than $80 \%$ of the collected cells were beating cardiomyocytes, and more than $99.99 \%$ of healthy cultivated cells were cardiomyocytes. The shrinkage and unattachment in our re-cultivation procedure might be caused by the insufficient removal of agarose coating of cultivation microchamber array from collagen layer. It should be noted that, although we did not count into the myosin-positive cells, even the shrinked cells in microchambers showed the positive-staining (see the white single arrowhead in Figure 5c).

In conclusion, we have succeeded in separating cardiomyocytes with label-free on-chip imaging cell sorting procedures with using of the index of their surface roughness, and the results indicated that the more than $80 \%$ of the smooth cells were beating cardiomyocyte cells, and also more than $99 \%$ of healthy re-cultivated cells were cardiac myosin positive cells. The results suggested that the non-labeling simple phase-contrast image-based cell sorting has a potential to separate cardiomyocytes precisely from the mixture of heart cells by the index of cell surface roughness with less than $1 \%$ error rate.

\section{Acknowledgements} NEDO).

This work was financially supported by New Energy Development Organization

\section{References}

1. Manabe I, Shindo T, Nagai R (2002) Gene expression in fibroblasts and fibrosis. Circ Res 91: 1103-1113. 
Citation: Nomura F, Kaneko T, Hattori A, Yasuda K (2011) Label-Free Shape-Based Selection of Cardiomyocytes with on-Chip Imaging Cell Sorting System. J Bioprocess Biotechniq S3:003 doi:10.4172/2155-9821.S3-003

2. Borg TK, Rubin K, Lundgren E, Borg K, Obrink B (1984) Recognition of extracellular matrix components by neonatal and adult cardiac myocytes. Dev Biol 104: 86-96.

3. Bonner WA, Hulett HR, Sweet RG, Herzenberg LA (1972) Fluorescence activated cell sorting. Rev Sci Instrum 43: 404-409.

4. Takahashi K, Hattori A, Suzuki I, Ichiki T, Yasuda K (2004) Non-destructive on-chip cell sorting system with real-time microscopic image processing. J Nanobiotechnol 2: 5 .

5. Hattori A, Yasuda K (2010) Comprehensive study of microgel electrode for onchip electrophoretic cell sorting. Jpn. JAppl Phys 49: 06GM04-1-4.

6. Kojima K, Kaneko T, Yasuda K (2006) Role of the community effect of cardiomyocyte in the entrainment and reestablishment of stable beating rhythms. Biochem Biophys Res Commun 351: 209-215.

7. Moriguchi H, Wakamoto $\mathrm{Y}$, Sugio $\mathrm{Y}$, Takahashi K, Inoue I, et al. ( 2002) An agar-microchamber cell-cultivation system: flexible change of microchamber shapes during cultivation by photo-thermal etching. Lab Chip 2: 125-132.

8. Kojima K, Moriguchi H, Hattori A, Kaneko T, Yasuda K (2003) Two-dimensiona network formation of cardiac myocytes in agar microculture chip with $1480 \mathrm{~nm}$ infrared laser photo-thermal etching. Lab Chip 3: 292-296.

9. Kojima K, Kaneko T, Yasuda K (2005) Stability of beating frequency in cardiac myocytes by their community effect measured by agarose microchamber chip. J Nanobiotech 3: 4

10. Kaneko T, Kojima K, Yasuda K (2007) An on-chip cardiomyocyte cell network assay for stable drug screening regarding community effect of cell network size. Analyst 132: 892-898.

11. KanekoT, Nomura F, Yasuda K (2011) On-chip constructive cell-network study (I): contribution of cardiac fibroblasts to cardiomyocyte beating synchronization and community effect. J Nanobiotech 9: 21.
This article was originally published in a special issue, Cellomics and Tissueomics handled by Editor(s). Dr. Masamichi Kamihira, Kyushu University, Japan. 